\title{
Efecto de la edad sobre la percepción de los factores de riesgo de los trastornos de la conducta alimentaria en jugadoras senior profesionales de voleibol
}

\author{
Effect of age on perception of eating disorder risk \\ factors in professional volleyball players
}

\author{
Frideres, J.E. ${ }^{1}$, Visiedo, A. ${ }^{2}$ y Palao, J.M. ${ }^{3}$ \\ 1 Máster en Kinesiology, 2 Alumna Tercer ciclo. Universidad de Murcia, Murcia (España). \\ 3 Departamento de Actividad Física y Deporte. Universidad de Murcia, Murcia. (España). \\ Recibido: 03/03/2014; Aceptado:14/10/2014
}

\begin{abstract}
Resumen: El objetivo del estudio fue conocer la percepción de las jugadoras profesionales de voleibol en relación al control del peso, nutrición, y riesgo de trastornos de la conducta alimentaria en función de su edad. La muestra objeto de estudio estuvo compuesta por 41 deportistas de siete equipos de voleibol de categoría senior (primera división nacional). Se realizó un diseño selectivo, descriptivo, y transversal. La muestra fue dividida en dos grupos en función de su edad, jóvenes y mayores, para su análisis. Se empleó un cuestionario para obtener la información. La percepción de las deportistas en relación al control del peso, nutrición, y riesgo de trastornos de la conducta alimentaria en función de la edad de las jugadoras senior profesionales no varía en función de su edad. Los resultados muestran que el rol del entrenador como fuente de información para las jugadoras ha cambiado con respecto a las de menor edad. El trabajo plantea la necesidad de adoptar planes de actuación pro-activos para minimizar el riesgo de TCA en el deporte del voleibol.

Palabras clave: deporte, rendimiento, percepción, nutrición
\end{abstract}

Abstract: The purpose of this study was to assess professional female vol leyball players' attitudes toward weight control, diet, and eating disorders with regard to their age. The sample consisted of 41 players from seven teams of the Spanish first division that competed in the National Club Cup. A descriptive, selective, and transversal design was used. The sample was divided into younger and older players for its analysis. The information was obtained from a survey. Players' attitudes toward weight control, diet, and eating disorders did not change with regard to their age. The studied players perceived that they had enough knowledge about nutrition and eating disorders. The results show that the coach's role as a source of information for athletes has changed. The paper proposes the need to adopt pro-active strategies to minimize the risk of eating disorders in volleyball.

Keywords: sport, performance, self-perception, nutrition.

\section{Introducción}

El entrenamiento busca preparar al deportista para la competición. Con el entrenamiento se intentan mejorar o controlar todos los aspectos que teóricamente afectan al rendimiento. Dentro de este proceso, en voleibol femenino, es habitual controlar el peso de las jugadoras. A través de ese control, se busca maximizar el trabajo de fuerza (altura de salto en el remate y en el bloqueo), al mismo tiempo que se busca reducir el riesgo de lesión (sobrecarga de las articulaciones en el salto) (Reeser et al, 2006). Este énfasis en el control del peso y de la forma deportiva se ve incrementado por la implicación en el proceso de los entrenadores y las propias jugadoras (Becker, 1992; Khan y Bahr, 2003). Esto hace que las deportistas tengan que ser capaces de soportar estrés tanto a nivel físico-biológico (tipo, volumen, e intensidad de los entrenamientos, etc.) como a nivel psicológico (auto-presión del propio deportista, presión del entrenador y/o compañeros, público, medios de comunicación, etc.) (American College

Dirección para correspondencia [Correspodence address]: Jillian E. Frideres. Avd. Reina Sofía, 32. Guadalupe, 30107, Murcia (España). E-Mail: jefrideres@gmail.com of Sports Medicine, 1997; Urhausen, Gabriel, y Kindermann, 1995; Williams y Andersen, 1998). El control del peso y su énfasis incide directamente en el riesgo de padecer trastornos de la conducta alimentaria (TCA) por parte de las jugadoras (Stice, 2002; Striegel-Moore y Bulik, 2007; Rhea, 1998).

En el deporte profesional, el estrés que las deportistas soportan se ve incrementado por el seguimiento que los medios de comunicación hacen sobre éstas (Davis y Tuggle, 2012) y por las exigencias reglamentarias de tener que llevar ropa ajustada para competir (Regla 4.3, FIVB, 2004) (Rust, 2002). Las investigaciones, tanto en la población general como en el deporte en general y en voleibol de forma específica, muestran que la edad donde el riesgo de padecer TCA es mayor es en la adolescencia (Stice, 2002; Striegel-Moore y Bulik, 2007). Así, aunque las jugadoras de mayor nivel de competición soportan mayor estrés en relación al control del peso, éstas tienen mayor capacidad y recursos de soportar el estrés que las jóvenes deportistas (Frideres y Palao, 2005a, 2005b). Esta tendencia se ha observado en atletas en formación, pero no se ha estudiado en jugadoras formadas. Los estudios revisados en la bibliografía no han profundizado si en deportistas 
formadas también se da esta tendencia, a mayor edad mayor habilidad para soportar el estrés. Estos estudios muestran que el conocimiento sobre nutrición y TCA que las jugadoras poseen no es adecuado y que es recomendable la realización de programas formativos específicos (Torres-McGehee, et al., 2012; Valliant, Emplaincourt, Wenzel, y Garner, 2012).

El entrenamiento no solo debe buscar incrementar el rendimiento, sino también debe velar porque la salud de los deportistas no se vea afectada. En el tratamiento de los TCA, la formación y la reducción de las situaciones de riesgo se consideran la medida más adecuada para lograr reducir su incidencia (Beals, et al., 1999; Papanek, 2003). El presente trabajo busca conocer en qué situación se encuentran las jugadoras profesionales de voleibol y su percepción de la situación en función de su madurez y experiencia (edad). Estudios previos indican que esta variable puede influir en la capacidad de soportar estrés y en el conocimiento que tienen las deportistas. El presente trabajo busca verificar esta hipótesis, que cuanto mayor es la experiencia de las jugadoras, mayor es su capacidad de soportar estrés y su conocimiento. El objetivo del estudio fue conocer la percepción de las jugadoras profesionales de voleibol en relación al control del peso, nutrición, y riesgo de trastornos de la conducta alimentaria en función de su edad.

\section{Método}

\section{Participantes}

La muestra objeto de estudio estuvo compuesta por 41 jugadoras de siete equipos de voleibol de categoría senior (primera división nacional) participantes en la Copa de la Reina (año 2006-2007). La muestra fue dividida en dos grupos en función de su edad, jóvenes (17.8 - 23.0 años, $\mathrm{n}=22)$ y mayores (23.1 32.3 ańos, $n=19)$. Las características de la muestra se presentan en la tabla 1. La participación en el estudio fue voluntaria. Previamente a la administración de los cuestionarios, las responsables de los equipos y de las jugadoras fueron informadas del objetivo del trabajo y cumplimentaron el correspondiente consentimiento informado. Dos equipos participantes en el campeonato no estuvieron interesados en participar en el estudio.

Tabla 1. Características de las jugadoras profesionales de voleibol objeto de estudio.

\begin{tabular}{llllllll}
\hline & $\begin{array}{l}\text { Jugadoras } \\
\text { jóvenes }\end{array}$ & \multicolumn{3}{l}{$\begin{array}{l}\text { Jugadoras } \\
\text { mayores }\end{array}$} & \multicolumn{3}{l}{ Totales } \\
& $\mathrm{M}$ & $\mathrm{DT}$ & $\mathrm{M}$ & $\mathrm{DT}$ & $\mathrm{M}$ & $\mathrm{DT}$ \\
\hline Edad & 21.57 & 1.57 & 26.29 & 1.86 & 23.68 & 2.91 \\
Altura & 1.78 & .06 & 1.78 & .08 & 1.78 & .07 \\
Peso & 69.62 & 7.15 & 68.47 & 6.90 & 69.11 & 6.97 \\
$\begin{array}{l}\text { Horas diarias } \\
\text { entrenamiento }\end{array}$ & 3.63 & .98 & 3.38 & 1.14 & 3.52 & 1.04 \\
\hline
\end{tabular}

\section{Procedimiento}

Las variables de estudio fueron: motivos de practicar voleibol, nivel de satisfacción con su peso, la frecuencia y forma de control del peso, personas que influyen en el control del peso, motivos para controlar el peso, percepción sobre la adecuación de su dieta, información sobre nutrición y trastornos de la conducta alimentaria, fuentes de información, énfasis del entrenador y compañeras en el control de peso, si alguien les ha expresado preocupación por sus patrones alimenticios y su actitud ante su peso, y si creen que hay problemas de control del peso en el voleibol. La información fue obtenida mediante un cuestionario. Los cuestionarios fueron entregados a las jugadoras por uno de los investigadores, y fueron cumplimentados por éstas al principio del campeonato.

\section{Instrumental}

La información respecto a la percepción de las deportistas se obtuvo mediante el cuestionario "Alimentación, control de peso, y deporte" (Frideres y Palao, 2005a). El cuestionario estaba compuesto por 10 preguntas cerradas y cinco preguntas semi-abiertas. La validez de contenido fue establecida por expertos y el coeficiente de fiabilidad más bajo de las preguntas del cuestionario era de 0.94 (Frideres y Palao, 2005a).

\section{Estadística}

Se realizó un análisis descriptivo (medias, desviaciones típicas, y porcentajes) e inferencial (test de Chi-cuadrado y tablas de contingencia) para estudiar las diferencias en función de la edad en las variables estudias. El análisis estadístico se realizó empleando el software SPSS 20.0. El nivel de significación se estableció en $\mathrm{p}<.05$.

\section{Resultados}

No se encontraron diferencias significativas en ninguna de las variables relativas a la motivación, satisfacción y control del peso en relación a la edad de las deportistas (Tabla 2). Se encontraron diferencias significativas en una de las fuentes de información sobre la dieta (amigos) (Tabla 3). En el resto de variables estudiadas no se encontraron diferencias entre las jugadoras jóvenes y mayores (percepciones relativas a la dieta y TCA). 
Tabla 2. Motivos de práctica, satisfacción del peso, y control del peso en jugadoras profesionales de voleibol en función de su edad.

\begin{tabular}{|c|c|c|c|c|c|c|c|}
\hline & \multicolumn{2}{|c|}{ Jugadoras jóvenes } & \multicolumn{2}{|c|}{ Jugadoras mayores } & \multirow{2}{*}{$p$} & \multicolumn{2}{|c|}{ Totales } \\
\hline & $\mathrm{n}$ & $\%$ & $\mathrm{n}$ & $\%$ & & $\mathrm{n}$ & $\%$ \\
\hline \multicolumn{8}{|l|}{ Motivos practica voleibol } \\
\hline Realizar ejercicio físico & 13 & 59.1 & 7 & 36.8 & & 20 & 48.8 \\
\hline Salud & 9 & 40.9 & 8 & 42.1 & & 17 & 41.5 \\
\hline Diversión & 18 & 81.8 & 12 & 63.1 & & 30 & 73.2 \\
\hline Amigos & 4 & 18.2 & 2 & 10.5 & & 6 & 14.6 \\
\hline Trabajo & 14 & 63.6 & 16 & 84.2 & & 30 & 73.2 \\
\hline Viajar & 3 & 13.6 & 2 & 10.5 & & 5 & 12.2 \\
\hline Propio deporte & 10 & 45.4 & 8 & 42.1 & & 18 & 43.9 \\
\hline Estar en forma & 6 & 27.3 & 8 & 47.1 & & 14 & 34.1 \\
\hline Sentirse bien & 3 & 13.6 & 1 & 5.3 & & 4 & 9.8 \\
\hline Competir & 8 & 36.4 & 7 & 36.8 & & 15 & 36.6 \\
\hline Otros (desarrollo personal) & 8 & 36.4 & 5 & 26.3 & & 13 & 31.7 \\
\hline \multicolumn{8}{|l|}{ Satisfacción peso } \\
\hline Totalmente satisfecha & 2 & 9.1 & 3 & 15.8 & & 5 & 12.2 \\
\hline Satisfecha & 11 & 50 & 12 & 63.2 & & 23 & 56.1 \\
\hline Poco satisfecha & 8 & 36.4 & 3 & 15.8 & & 11 & 26.8 \\
\hline Nada satisfecha & 1 & 4.5 & 0 & 0.0 & & 1 & 2.4 \\
\hline \multicolumn{8}{|l|}{ Frecuencia control del peso } \\
\hline No controla & 11 & 50 & 8 & 42.1 & & 19 & 46.3 \\
\hline $1-2$ veces al mes & 5 & 22.7 & 7 & 36.8 & & 12 & 29.3 \\
\hline $1-2$ veces a la semana & 4 & 18.2 & 4 & 21.1 & & 8 & 19.5 \\
\hline Todos los días & 2 & 9.1 & 0 & 0.0 & & 2 & 4.9 \\
\hline Control del peso y forma de control & & & & & & 0 & 0.0 \\
\hline Sí controlan el peso & 13 & 59.1 & 7 & 36.8 & & 20 & 48.8 \\
\hline Ayuno & 9 & 40.9 & 8 & 42.1 & & 17 & 41.5 \\
\hline Más entrenamiento & 18 & 81.8 & 12 & 63.2 & & 30 & 73.2 \\
\hline Retención de fluidos & 4 & 18.2 & 2 & 10.5 & & 6 & 14.6 \\
\hline Vómitos & 14 & 63.6 & 16 & 84.2 & & 30 & 73.2 \\
\hline Comida (cantidad) & 12 & 54.5 & 8 & 42.1 & & 20 & 48.8 \\
\hline Comida (calorías) & 6 & 27.3 & 9 & 47.4 & & 15 & 36.6 \\
\hline Laxantes & 3 & 13.6 & 1 & 5.3 & & 4 & 9.8 \\
\hline Píldoras dietéticas & 8 & 36.4 & 7 & 36.8 & & 15 & 36.6 \\
\hline Otros (tipos de comidas) & 5 & 22.7 & 1 & 5.3 & & 6 & 14.6 \\
\hline \multicolumn{8}{|l|}{ Influencia en el control del peso } \\
\hline Entrenador & 8 & 36.4 & 4 & 21.1 & & 12 & 29.3 \\
\hline Compañeras de equipos & 1 & 4.5 & 2 & 10.5 & & 3 & 7.3 \\
\hline Familiares y/o novio/a & 21 & 95.5 & 19 & 100.0 & & 40 & 97.6 \\
\hline Libros/revistas & 4 & 18.2 & 3 & 15.8 & & 7 & 17.1 \\
\hline Sociedad & 0 & 0 & 0 & 0.0 & & 0 & 0.0 \\
\hline Amigos & 1 & 4.5 & 0 & 0.0 & & 1 & 2.4 \\
\hline Médico & 1 & 4.5 & 0 & 0.0 & & 1 & 2.4 \\
\hline Televisión & 1 & 4.5 & 0 & 0.0 & & 1 & 2.4 \\
\hline Decisión personal & 4 & 18.2 & 2 & 10.5 & & 6 & 14.6 \\
\hline \multicolumn{8}{|c|}{ Creencia necesidad control del peso en voleibol } \\
\hline Exceso peso afecta al rendimiento & 12 & 54.5 & 8 & 42.1 & & 8 & 19.5 \\
\hline Exceso peso incrementa lesiones & 5 & 22.7 & 9 & 47.4 & & 9 & 22.0 \\
\hline \multicolumn{8}{|l|}{ Motivos para control del peso } \\
\hline Apariencia & 8 & 36.4 & 4 & 21.1 & & 4 & 9.8 \\
\hline Rendimiento & 11 & 50 & 10 & 52.5 & & 10 & 24.4 \\
\hline Salud & 7 & 31.8 & 10 & 52.5 & & 10 & 24.4 \\
\hline
\end{tabular}

Efecto de la edad sobre la percepción de los factores de riesgo de los trastornos de la conducta alimentaria en jugadoras senior profesionales de voleibol. SporTK, 3(1-2), 27-32 
Tabla 3. Adecuación, información, y percepciones relativas a la dieta, y TCA en jugadoras profesionales de voleibol en función de su edad.

\begin{tabular}{|c|c|c|c|c|c|c|c|}
\hline & \multicolumn{2}{|c|}{ Jugadoras jóvenes } & \multicolumn{2}{|c|}{ Jugadoras mayores } & \multirow{2}{*}{$p$} & \multicolumn{2}{|c|}{ Totales } \\
\hline & $\mathrm{n}$ & $\%$ & $\mathrm{n}$ & $\%$ & & $\mathrm{n}$ & $\%$ \\
\hline \multicolumn{8}{|c|}{ Adecuación dieta a edad y actividad física } \\
\hline Adecuada & 16 & 72.7 & 16 & 84.2 & & 32 & 78.0 \\
\hline No adecuada & 6 & 27.3 & 3 & 15.8 & & 9 & 22.0 \\
\hline \multicolumn{8}{|l|}{ Información sobre dieta } \\
\hline Mucha & 5 & 22.7 & 7 & 36.8 & & 12 & 29.3 \\
\hline Bastante & 6 & 27.3 & 9 & 47.4 & & 15 & 36.6 \\
\hline Suficiente & 9 & 40.9 & 2 & 10.5 & & 11 & 26.8 \\
\hline Insuficiente & 2 & 9.1 & 1 & 5.3 & & 3 & 7.3 \\
\hline \multicolumn{8}{|c|}{ Fuente de información sobre dieta } \\
\hline Entrenador & 8 & 36.4 & 5 & 29.4 & & 13 & 31.7 \\
\hline Compañeras de equipos & 7 & 31.8 & 3 & 17.6 & & 10 & 24.4 \\
\hline Familiares y/o novio/a & 3 & 13.6 & 0 & 0 & & 3 & 7.3 \\
\hline Libros/revistas & 14 & 63.6 & 11 & 57.9 & & 25 & \\
\hline Internet & 13 & 59.1 & 7 & 36.8 & & 20 & 48.8 \\
\hline Amigos & 7 & 31.8 & 1 & 5.9 & 0.047 & 8 & 19.5 \\
\hline Médico & 3 & 13.6 & 2 & 10.5 & & 8 & 19.5 \\
\hline Televisión & 2 & 9.1 & 0 & 0 & & 2 & 4.9 \\
\hline Otros (dietista) & 3 & 13.6 & 3 & 15.8 & & 6 & \\
\hline \multicolumn{8}{|c|}{ Información sobre TCA y fuente } \\
\hline Adecuada y suficiente & 20 & 90.9 & 16 & 84.2 & & 36 & 87.8 \\
\hline \multicolumn{8}{|c|}{ Fuente de información sobre TCA } \\
\hline Entrenador & 9 & 40.9 & 8 & 42.1 & & 17 & 41.5 \\
\hline Compañeras de equipos & 1 & 4.5 & 0 & 0 & & 1 & 2.4 \\
\hline Familiares y/o novio/a & 0 & 0 & 0 & 0 & & 0 & 0.0 \\
\hline Libros/revistas & 15 & 68.2 & 17 & 89.5 & & 32 & 78.0 \\
\hline Internet & 5 & 22.7 & 2 & 10.5 & & 7 & 17.1 \\
\hline Sistema educativo reglado & 15 & 68.2 & 9 & 47.4 & & 24 & 58.5 \\
\hline Médico & 3 & 13.6 & 2 & 10.5 & & 5 & 12.2 \\
\hline Televisión & 7 & 31.8 & 5 & 26.3 & & 12 & 29.3 \\
\hline \multicolumn{8}{|c|}{ Énfasis del entrenador control peso } \\
\hline Demasiado & 3 & 13.6 & 1 & 5.3 & & 4 & 9.8 \\
\hline Justo & 2 & 9.1 & 2 & 10.5 & & 4 & 9.8 \\
\hline No suficiente & 1 & 4.5 & 2 & 10.5 & & 3 & 7.3 \\
\hline No enfatiza & 17 & 77.2 & 14 & 73.7 & & 31 & 75.6 \\
\hline \multicolumn{8}{|c|}{ Énfasis de las compañeras control peso } \\
\hline Demasiado & 2 & 9.1 & 1 & 5.3 & & 3 & 7.3 \\
\hline Justo & 2 & 9.1 & 2 & 10.5 & & 4 & 9.8 \\
\hline No suficiente & 2 & 9.1 & 2 & 10.5 & & 4 & 9.8 \\
\hline No enfatiza & 16 & 72.7 & 14 & 73.7 & & 30 & 73.2 \\
\hline \multicolumn{8}{|c|}{ ¿Te han expresado otras personas preocupación por tus patrones alimenticios? } \\
\hline Sí & 8 & 36.4 & 6 & 31.6 & & 14 & 34.1 \\
\hline \multicolumn{8}{|c|}{ ¿Te han expresado otras personas preocupación por tu actitud ante tu peso? } \\
\hline Sí & 4 & 18.2 & 2 & 10.5 & & 6 & 14.6 \\
\hline \multicolumn{8}{|c|}{ ¿Crees que hay problema con el control del peso en voleibol? } \\
\hline Sí & 1 & 4.5 & 4 & 21.1 & & 5 & 12.2 \\
\hline
\end{tabular}

\section{Discusión}

El objetivo de este estudio fue conocer cuál es la percepción de las jugadoras de voleibol en relación al control del peso, nutrición, y riesgo de trastornos de la conducta alimentaria en función de la edad. Las participantes en el estudio eran deportistas de rendimiento en un deporte que requiere de técnica y táctica junto a unas cualidades físicas específicas para 
alcanzar el éxito deportivo. La necesidad de realizar saltos de forma continua y el uso de ropa ceñida son las principales causas para emplear técnicas de control del peso por parte de las jugadoras (Becker, 1992). Se parte de la hipótesis de la posibilidad de la existencia de diferencias en función de los años de experiencia. Así, a mayor número de vivencias, mejor capacidad para soportar el estrés.

A nivel general, los resultados encontrados muestran que la percepción y conocimientos de las jugadoras no varían en función de la edad (jóvenes vs. mayores). Estos resultados no confirman la hipótesis antes planteada, y muestran que se produce una estabilización en este grupo de edad y nivel. Teniendo en cuenta lo que ocurre en grupos de edad más bajos, los valores encontrados son mayores que los de categorías de formación (Frideres y Palao, 2005a). Esto muestra una evolución en escalera que se estabiliza en la edad senior. Estos datos parecen indicar que la consolidación del conocimiento, percepción y hábitos relativos a los TA se produce en categorías inferiores y en la primera fase del proceso de profesionalización.

A nivel de fuentes de información, se encontraron diferencias en función de la edad en la información que reciben las jugadoras jóvenes por parte de los amigos. Estos resultados pueden indicar que este es el entorno más influyente en las deportistas jóvenes. Esto puede deberse a que salen del entorno familiar. A este respecto, las deportistas de mayor edad tienen unos patrones de actuación ya consolidados y no se ven afectados por este entorno de la misma forma. En ambos grupos de edad, los datos encontrados indican tendencias similares y muestran una estabilización en su forma de proceder u obtener la información. En comparación con estudios previos, estos resultados muestran cambios en las principales fuentes de información de las cuales proceden los datos relativos a nutrición y dietas. Las fuentes de información son obtenidas directamente por las deportistas (libros e internet) y no provienen del entrenador, el nutricionista, o el médico.

Estos resultados difieren en parte de los encontrados en otros grupos de edad (Frideres y Palao, 2005a) y otros tipos de población. En jugadoras universitarias estadounidenses de voleibol, sí se ha encontrado que los entrenadores aportan información sobre nutrición a sus atletas (Jacobson, Sobonya, y Ranson, 2001). El rango de edad de estas deportistas universitarias corresponde con deportistas jóvenes en el presente trabajo. Se debe tener en cuenta que estos estudios se han realizado en un entorno educativo universitario y no profesional como en el presente trabajo más enfocado al rendimiento. Sin embargo, si se considera el aporte de información por parte de los entrenadores, se observa que estos aportan más información sobre TA en ambos grupos de edad que sobre nutrición. Estos pueden estar relacionado con un cambio en la sensibilización de los entrenadores con respecto a este tema. La estabilización en el conocimiento muestra que o bien los entrenadores no adaptan el aporte de información al conocimiento de las deportistas o bien que alcanzado un nivel, consideran que hay más aspectos en los que deben centrarse. La utilización de otros medios para obtener información por parte de las deportistas (internet, revistas y libros) muestra la necesidad de que la formación educativa de las jugadoras sea sistemática, planificada, y evolucione junto a la jugadora. Este proceso no puede depender de ellas.

Este aporte de información por parte de los entrenadores debe ser parte de un abordaje multi-disciplinar centrado en velar por la salud de los deportistas (Brown, 2013; Valliant, et al., 2012; Temme y Hoch, 2013). Si la prevención es la mejor forma de combatir esta enfermedad (Beals, et al., 1999; Papanek, 2003), el objetivo debe ser que el $100 \%$ de las deportistas tenga suficiente información. Esta formación teórico-práctica debe proporcionar conocimientos y capacidad de valorar la información sobre nutrición y control del peso que llega a las jugadoras de sus amigos o Internet. No se puede olvidar la facilidad de acceder a la información en la última década a través de internet (Torres-McGehee, et al., 2012). El objetivo de estos programas es evitar el riesgo de problemas para la salud y debe implicar tanto a atletas como a entrenadores y preparadores físicos (Almeida y Soares, 2003; Vargas, KerrPrietchett, Papadopoulous, y Bennett, 2013).

El conocimiento de la percepción de las jugadoras sobre los aspectos relativos al control del peso, nutrición, y riesgo de trastornos de la conducta alimentaria puede ayudar a conocer cuáles son las características de esta población, la situación de esta problemática, y planificar actuaciones al respecto para esta población. En esta línea, los resultados muestran que la muestra estudiada tiene una percepción de que su conocimiento a nivel de nutrición y TCA es elevado. Sin embargo, esta creencia de las jugadoras difiere con los resultados de estudios previos que valoraron su conocimiento y encontraron que éste no era adecuado (Torres-McGehee, et al., 2012; Valliant, Emplaincourt, Wenzel, y Garner, 2012). Esto hace necesario la realización de trabajos que estudien la relación entre estos dos aspectos (percepción y conocimiento real). Otro parte a resaltar es que el 30\% de la muestra no está satisfecha con su peso $(30 \%)$ y lo controla varias veces a la semana de forma regular $(-20 \%)$. Estas deportistas además emplean métodos y técnicas de pérdida de peso que en ocasiones no son adecuadas para su salud y rendimiento deportivo. El hecho de que las deportistas hagan uso de este tipo de métodos de control del peso, probablemente por una decisión personal, hace necesario la atención por parte de los entrenadores y especialistas sobre este tema. Estudios en otros deportes colectivos han mostrado la necesidad de la implementación de programas de formación para reducir la incidencia de este problema (Molina-López et al., 2013). 


\section{Conclusión}

La percepción de las jugadoras de voleibol en relación al control del peso, nutrición, y riesgo de trastornos de la conducta alimentaria en función de la edad de las jugadoras senior profesionales no varía en función de su edad. Los resultados muestran que en edad senior a mayor experiencia y vivencias no incrementa su capacidad de soportar estrés y su conocimiento. Los resultados muestran que el rol del entrenador como fuente de información ha cambiado y como se hace necesario adoptar planes de actuación pro-activos para minimizar el riesgo de TCA en el deporte del voleibol. Se hace necesario la implementación de programas de formación relativos a nutrición y TA para las jugadoras y entrenadores.

\section{Referencias}

1. Almeida, T.A., y Soares, E. A. (2003). Nutritional and anthropometric profile of adolescent volleyball athletes. Revista Brasileira de Medicina do Esporte, 9(4), 198-203.

2. American College of Sports Medicine (1997). The female athlete triad. Medicine and Science in Sports and Exercise, 29, 1-9.

3. Beals, K. A., Brey, R. A., y Gonyou, J. B. (1999). Understanding the female athlete triad: Eating disorders, amenorrhea, and osteoporosis. The Journal of School Health, 69(8), 337-340.

4. Becker, B. (1992). The effects of experimental alterations in excess weight on vertical jump performance. Unpublished master's thesis, University of Northern Iowa, Cedar Falls, IA, USA

5. Brown, K. N. (2013). Nutrition education to minimize health risk: Approaches for teaching college students and female high school athletes. Unpublished doctoral dissertation. Utah State University, Logan, UT, USA.

6. Cortez, D., Krebs-Holm, L., Gish, D., y Wildman, R. (2011). Anthropometric measures and nutrition intake, habits and perceptions of Division I women's volleyball players. Journal of the International Society of Sports Nutrition, 8(Supple 1), P8.

7. Davis, K. K., y Tuggle, C. A. (2012). A gender analysis of NBC's coverage of the 2008 summer Olympics. Electronic News, 6(2), 51-66.

8. FIVB (2004). Reglas oficiales de voleibol 2004-2008. Lausane. FIVB.

9. Frideres, J. E., y Palao, J. M. (2004). Eating disorders among division III female cross-country runners. IAHPERD Journal, 37(1), 15-19.

10. Frideres, J. E., y Palao, J. M. (2005a). Eating disorder risk factors perceived by adolescent female volleyball players. International Journal of Volleyball Research, 8(1), 24-28.

11. Frideres, J. E., y Palao, J. M. (2005b). Estudio descriptivo de los factores de riesgo de los trastornos alimenticios en atletas universitarias de cross country. Lecturas: Educación Física y Deportes, 89, 1-8.

12. Frideres, J. E., y Palao, J. M. (2008a). Percepción de los factores de riesgo de los trastornos alimenticios en jugadoras universitarias de voleibol. Cuadernos de Psicología del Deporte, 8(1), 94.

13. Frideres, J. E., y Palao, J. M. (2008b). Percepción de los factores de riesgo de los trastornos alimenticios en jugadoras de baloncesto, fútbol sala y voleibol. Lecturas: Educación Física y Deportes, 125, 1-15.

14. Hulley, A. J., y Hill, A. J. (2001). Eating disorders and health in elite women distance runners. International Journal of Eating Disorders, 30(3), 312-317.

15. Jacobson, B. H., Sobonya, C., y Ransone, J. (2001). Nutrition practices and knowledge of college varsity athletes: A follow-up. The Journal of Strength and Conditioning Research, 15(1), 63-68.

16. Khan, K., y Roald B. (2003). Knee and ankle injuries in volleyball (pp.
130-140). En J. C. Reeser y R. Bahr (Eds), Handbook of Sports Medicine and Science: Volleyball. Londres: John Wiley and Sons.

17. Molina-López, J., Molina, J. M., Chirosa, L. J., Florea, D., Sáez, L., Jiménez, J., Planells, P., Pérez de la Cruz, A., y Planells, E. (2013). Implementation of a nutrition education program in a handball team; consequences on nutritional status. Nutrición Hospitalaria, 28(4), 1065-1076.

18. Papanek, P. E. (2003). The female athlete triad: An emerging role for physical therapy. Journal of Orthopaedic and Sport Physical Therapy, 33(10), 594-614.

19. Reeser, J. C., Verhagen, E. A. L. M., Briner, W. W., Askeland, T. I., y Bahr, R. (2006). Strategies for the prevention of volleyball related injuries. British Journal of Sports Medicine, 40(7), 594-600.

20. Rhea, D. (1998). Eating disorders: Ethnic differences of volleyball players. USA Volleyball Sports Medicine and Performance Commission. Recuperado julio 2003 de http://www.avca.org/sportsmed/smpcArticles/ smpcEating.htm

21. Rust, D. M. (2002). The female athlete triad: Disordered eating, amenorrhea, and osteoporosis. The Clearing House, 75(6), 301-305.

22. Stice, E. (2002). Risk and maintenance factors for eating pathology: A meta-analytic review. Psychological Bulletin, 128(5), 825.

23. Striegel-Moore, R. H., y Bulik, C. M. (2007). Risk factors for eating disorders. American Psychologist, 62(3), 181.

24. Temme, K. E., y Hoch, A. Z. (2013). Recognition and rehabilitation of the female athlete triad/tetrad: A multidisciplinary approach. Current Sports Medicine Reports, 12(3), 190-199.

25. Torres-McGehee, T. M., Pritchett, K. L., Zippel, D., Minton, D. M. Cellamare, A., y Sibilia, M. (2012). Sports nutrition knowledge among collegiate athletes, coaches, athletic trainers, and strength and conditioning specialists. Journal of Athletic Training, 47(2), 205-211.

26. Urhausen, A., Gabriel, H., y Kindermann, W. (1995). Blood hormones as markers of training stress and overtraining. Sports Medicine, 20(4), 251-276.

27. Valliant, M. W., Pittman Emplaincourt, H., Kieckhaefer Wenzel, R., y Garner, B. H. (2012). Nutrition education by a registered dietitian improves dietary intake and nutrition knowledge of a NCAA female volleyball team. Nutrients, 4, 506-516.

28. Vargas, S. L., Kerr-Pritchett, K., Papadopoulous, C., y Bennett, V. (2013). Dietary habits, menstrual health, body composition, and eating disorder risk among collegiate volleyball players: A descriptive study. International Journal of Exercise Science, 6(1), Article 7.

29. Williams, J. M., y Andersen, M. B. (1998). Psychosocial antecedents of sport injury: Review and critique of the stress and injury model. Journal of Applied Sport Psychology, 10(1), 5-25. 